\title{
Predicting residual rectal adenocarcinoma in the surgical specimen after preoperative brachytherapy with endoscopic ultrasound
}

\author{
Joseph Romagnuolo MD FRCPC MSc(Epid) ${ }^{1}$, Josée Parent MD FRCPC ${ }^{2}$, Té Vuong MD FRCPC ${ }^{3}$, \\ Mélanie Bélanger MD FRCPC ${ }^{2}$, René P Michel MD FRCPC ${ }^{4}$, Paul J Belliveau MD FRCSC ${ }^{5}$, Judith L Trudel MD FRCSC 5
}

\begin{abstract}
J Romagnuolo, J Parent, T Vuong, et al. Predicting residual rectal adenocarcinoma in the surgical specimen after preoperative brachytherapy with endoscopic ultrasound. Can J Gastroenterol 2004;18(7):435-440.
\end{abstract}

BACKGROUND AND STUDY AIMS: A novel brachytherapy (BT) protocol evaluated at McGill University has shown promise in terms of downstaging and achieving high tumour sterilization rates in rectal cancer. Endoscopic ultrasound (EUS) has emerged as the imaging modality of choice for local staging of rectal cancer. However, external beam radiotherapy appears to decrease the accuracy of EUS from $85 \%$ to $40 \%$. The aim of the present study was to prospectively evaluate the accuracy of EUS in assessing the response of rectal cancer to BT.

PATIENTS AND METHODS: Thirty-three patients with locally advanced (stage T2 or T3) operable rectal carcinomas were included in an experimental protocol involving a novel conformal technique, using three-dimensional planning, to administer high-dose rate preoperative BT. The 18 patients who were able to have a post-BT EUS exam arranged within two weeks before surgery (eg, four to eight weeks post-BT) were included in this study. Tumour (T)- and lymph node $(\mathrm{N})$-staging on radial EUS, as well as interpretation of the residual tumour, were assessed prospectively. Pathologists were blinded to the post-BT EUS results.

RESULTS: The mean age was 70 years $(S D \pm 11$; range, 52 to 93 years) and $78 \%$ of the patients were male. Pre-BT EUS indicated that 16 patients $(89 \%)$ were stage T3, and two were stage T2. Five patients (28\%) had positive nodes (N1) by ultrasound. With BT, the mean maximal wall thickness on EUS decreased from $14 \mathrm{~mm}$ to $9.4 \mathrm{~mm}(\mathrm{P}<0.001)$. At the time of surgery, seven of the 18 patients (39\%) had no detectable tumour in the resected specimen; one had carcinoma in situ, one was stage $\mathrm{T} 1$, one was stage $\mathrm{T} 2$, and eight were stage T3. Eleven patients (61\%) underwent an abdominoperineal resection, including four of the $11(36 \%)$ with no ultimate evidence of residual carcinoma. Eight patients (44\%) were node-positive. The sensitivity, specificity, and positive and negative predictive values of post-BT EUS in predicting residual tumour were $82 \%, 29 \%, 64 \%$ and $50 \%$, respectively. The post-BT EUS accurately predicted the T-stage in eight (44\%) patients; most errors were due to overstaging.

CONCLUSIONS: Rectal cancer T-staging by EUS post-BT is inaccurate, and although it appears sensitive in predicting the presence or absence of residual tumor in rectal adenocarcinoma after preoperative BT, the low predictive values in this setting limit its utility at this time.

Key Words: Brachytherapy; Endoscopic ultrasound; Rectal cancer; Tumour sterilization

\section{Prévisions, à l'échographie endoscopique, d'adénocarcinome rectal résiduel dans des prélèvements chirurgicaux après une curiethérapie préopératoire}

CONTEXTE ET OBJECTIF : Un nouveau protocole de curiethérapie,
évalué à l'Université McGill, s'est révélé prometteur quant à ses capacités
de faire régresser des tumeurs cancéreuses rectales et d'atteindre des taux
élevés de stérilisation. L'échographie endoscopique (EE) s'est avérée la
meilleure technique d'imagerie pour la stadification locale du cancer du
rectum. Toutefois, la radiothérapie externe semble diminuer la précision
de l'EE, qui la ferait passer de $85 \%$ à $40 \%$. La présente étude avait pour
but d'évaluer de façon prospective la précision de l'EE en déterminant la
réaction du cancer du rectum à la curiethérapie. PATIENTS ET MÉTHODE : Trente-trois patients atteints d'un cancer du rectum local, évolué (T2 ou T3) mais résécable ont participé au protocole expérimental qui faisait appel à une nouvelle technique d'imagerie conformationnelle, en trois dimensions, en vue d'être soumis à la curiethérapie à forte dose en phase préopératoire. Sur ce nombre, 18 ont pu subir une EE postcuriethérapie dans les deux semaines précédant la chirurgie, soit quatre à huit semaines après le traitement; ces derniers patients ont formé le groupe à l'étude. Il y a eu évaluation prospective du degré d'évolution de la tumeur $(\mathrm{T})$ et du degré d'atteinte des ganglions lymphatiques $(\mathrm{N})$ à l'EE radiale, de même qu'interprétation de la tumeur résiduelle. Les pathologistes ont été tenus dans l'ignorance des résultats de l'EE post-curiethérapie.

RÉSULTATS : Lâge moyen s'établissait à 70 ans (ET 11 [écart type]; plage : 52 à 93 ans) et $78 \%$ des patients étaient des hommes. Selon les résultats de l'EE pré-curiethérapie, 16 patients (89\%) étaient rendus au stade T3 et deux, au stade T2. L'échographie a révélé une atteinte des ganglions lymphatiques (N1) chez cinq patients (28\%). L'épaisseur maximale moyenne de la paroi à l'EE est passée de $14 \mathrm{~mm}$ à $9,4 \mathrm{~mm}(\mathrm{P}<0,001)$ avec la curiethérapie. Aucune trace de cancer n'a été décelée dans le prélèvement chirurgical chez 7 (39\%) des 18 patients, un cancer in situ chez un patient, un cancer $\mathrm{T} 1 \mathrm{chez}$ un patient, un cancer $\mathrm{T} 2 \mathrm{chez}$ un patient et un cancer T3 chez huit patients. Onze patients (61\%) ont subi une résection abdomino-périnéale, dont quatre (36\%) parmi les onze qui ne montraient pas de signe final de carcinome résiduel. Il y a eu envahissement des ganglions lymphatiques chez huit patients (44\%). La sensibilité, la spécificité et les valeurs prédictives positives et négatives de l'EE post-curiethérapie à l'égard des probabilités de tumeur résiduelle s'établissaient à $82 \%, 29 \%$, $64 \%$ et $50 \%$ respectivement. Le stade T a été évalué correctement à l'EE post-curiethérapie chez huit patients (44\%); la plupart des erreurs se sont soldées par une surévaluation du degré d'évolution.

CONCLUSION : L'EE post-curiethérapie ne permet pas une stadification satisfaisante du cancer du rectum et, même si la technique se montre sensible pour prévoir la présence ou l'absence de tumeur résiduelle dans les cas d'adénocarcinome rectal après une période de curiethérapie préopératoire, ses faibles valeurs prédictives dans le présent contexte en limitent actuellement l'utilité.

${ }^{1}$ Division of Gastroenterology and Department of Community Health Science, University of Calgary, Calgary, Alberta; ${ }^{2}$ Division of

Gastroenterology, ${ }^{3}$ Department of Radiation Oncology, ${ }^{4}$ Department of Pathology and ${ }^{5}$ Department of Surgery, McGill University Health

Centre, Montreal, Quebec

Correspondence: Dr Joseph Romagnuolo, University of Calgary Medical Clinic, 3350 Hospital Drive Northwest, Room G-179, Calgary, Alberta

T2N 4N1. Telephone 403-210-8575, fax 403-210-9368, e-mail j.romagnuolo@ucalgary.ca

Received for publication October 16, 2003. Accepted April 6, 2004 
$\mathrm{C}$ ancer of the colon and rectum is diagnosed in over 100,000 new patients each year in the United States (1). The surgical therapy for rectal adenocarcinoma can be particularly disabling to patients, because it involves abdominoperineal resection (APR) for cases in which a $2 \mathrm{~cm}$ distal tumour-free margin is not felt to be possible using an anterior approach. Selective pre- or postoperative chemoradiotherapy has traditionally been regarded as adjuvant, in an attempt to reduce local recurrence and perhaps improve survival, but it has not been intended to achieve cure as primary therapy $(2,3)$. Although endoluminal radiotherapy has been in use for over 20 years, this modality is still generally reserved for the small-sized stage $\mathrm{T} 1$ or perhaps $\mathrm{T} 2$ tumours and/or patients who are medically unfit for surgery (4-7). Curative brachytherapy (BT) with avoidance of surgical morbidity, along with sphincter-preservation, is an attractive theoretical option for small tumours $(8,9)$. Aggressive multimodality therapy for esophageal cancer, boasting high tumour sterilization (eg, absence of residual tumour) rates, has raised questions regarding the need for surgical therapy in patients with gastrointestinal cancer who have no residual tumour after chemoradiotherapy (10-12). The key, however, will be the accurate nonoperative identification of those patients.

Endoluminal ultrasound has been used since the early 1980s to stage rectal adenocarcinoma. In 14 studies reviewed by Heriot et al (13), many of which involved a nonoptical probe, this modality demonstrated a tumour $(\mathrm{T})$-staging accuracy of $75 \%$ to $93 \%$ (except for one study showing an accuracy of $67 \%$ ), and a lymph node (N)-staging accuracy of $77 \%$ to $88 \%$ (except for one study showing an accuracy of 62\%). Endoscopic ultrasound (EUS) also appears to be marginally more accurate than $\mathrm{T}$ - and $\mathrm{N}$-staging by pelvic magnetic resonance imaging (MRI) (14). It appears, however, that the accuracy of EUS T-staging can be reduced from $86 \%$ to $47 \%$ by external beam radiotherapy (15). This phenomenon has also been noted in esophageal cancer $(16,17)$. In these studies, although T-staging accuracy was reduced (17), sensitivity in predicting residual tumour was as high as $91 \%$, with a false positive rate of $12 \%(16)$.

McGill University Health Centre's novel preoperative conformal high-dose rate endoluminal BT protocol, using threedimensional computed tomography (CT)-simulator planning, has been associated with a complete clinical response rate as high as $65 \%$ in stage T2 and T3 tumours (18). This technique's high preoperative sterilization rate shows promise in avoiding APR, or surgery altogether, in patients without residual carcinoma. This depends, however, on the accuracy of EUS to predict the presence or absence of residual tumour after BT, which has not been assessed previously. Thus, the aim of the present study was to evaluate the sensitivity and specificity of EUS performed following a full course of endorectal high-dose rate BT in diagnosing residual carcinoma.

\section{METHODS}

Between October 1998 and October 2000, 33 patients were recruited into the study protocol to assess the feasibility and effectiveness of a novel conformal high-dose rate BT protocol for locally invasive rectal adenocarcinoma. Local institutional review board approval was obtained and all patients gave informed consent.

\section{Patient population}

Patients with newly diagnosed, locally advanced (stage T2 or T3) rectal adenocarcinoma who presented to the McGill University Health Centre and fulfilled the entry criteria, were recruited for the study protocol. Preoperative, pre-BT staging was performed by pelvic MRI, helical CT and rectal EUS (GF UM 30; Olympus Co, USA).

The study exclusion criteria were the following: inability to undergo surgical resection; stage T1 or T4 disease by either EUS or pelvic MRI; and evidence of distant metastases on helical CT. Ten per cent of patients met the exclusion criteria. Eighteen of the 33 patients recruited into the trial were able to have a preoperative and post-BT EUS arranged and performed within two weeks of the planned surgical resection; a post-BT EUS was logistically not able to be arranged in the narrow window between four and eight weeks post-BT and the surgical date for the others.

\section{BT protocol}

All patients were examined by flexible sigmoidoscopy and rectal EUS after completion of their MRI and CT; a pre-BT clinical staging was assigned using the American Joint Committee on Cancer TNM classification (19). During this examination, after the EUS was completed, endoclips (Olympus Co, USA) were endoscopically placed at the proximal and distal margins of the tumour mass to facilitate BT planning and imaging whenever technically possible.

Patients were treated with remote afterloading high-dose rate BT with a flexible multi-channel endorectal applicator (Nova applicator, Nucletron, The Netherlands), using a conformal technique involving three-dimensional CT-guided treatment planning, with the help of the endoscopically placed radiopaque endorectal clips. Dosimetry was tailored to the radial position of the bulk of the tumour, with differential loading of the delivery catheters. A dose of 26 Gy in four consecutive fractions (10 min to $20 \mathrm{~min}$ ) of $6.5 \mathrm{~Gy}$ was delivered to the tumour radial margin. Conformal dosimetry was achievable for tumours up to $15 \mathrm{~cm}$ from the anal verge and at a maximum depth of $3 \mathrm{~cm}$. Four to eight weeks were allowed for tumour downstaging before surgical resection.

\section{EUS}

Patients underwent a repeat EUS examination, performed by a single dedicated endosonographer, six weeks (range four to eight weeks) after completion of BT, and within two weeks before the planned surgical resection. The endosonographer was not blinded to the pre-BT EUS results. Patients with mild circumferential thickening of all wall layers, with layer blurring but without a focal hypoechoic mass, were considered to have inflammatory changes but no residual tumour; those thought to have residual tumour were re-staged according the American Joint Committee on Cancer (TNM) classification (19). Allowance was made for the frequent mildly irregular appearance of the serosal margin endosonographically, which was felt to represent radiation-related inflammatory change. Therefore, a diagnosis of a stage T3 lesion after BT was made only when the serosal margin appeared frankly invaded by a definite mass or obvious pseudopod projections. The maximal wall thickness (MWT) was also recorded for each patient. Nodal status was determined by accepted endosonographic criteria (node size greater than $1.0 \mathrm{~cm}$, hypoechogenicity, round shape and discrete margins). 
Surgery and pathological examination

All patients underwent surgical resection, based on pre-BT staging alone. APR was planned if it was felt by the surgeon that a $2 \mathrm{~cm}$ distal tumour-free margin was not achievable with low anterior resection. All of the resected surgical specimens were examined by one of the authors (RPM) and pathological staging was assessed using the American Joint Committee on Cancer TNM classification (19). Examination of the specimen was carried out without knowledge of the post-BT EUS results.

The resected colorectal specimens were fixed in $10 \%$ buffered formalin for one to three days, then described and sectioned according to a uniform protocol: multiple longitudinal $5 \mathrm{~mm}$ thick slices were made throughout the abnormal areas, either ulcerated or containing macroscopically visible tumour, and multiple tissue blocks were taken from all of these areas; in most cases, the entire abnormal-appearing regions were submitted for histological examination. The sections were embedded in paraffin, processed using standard histological techniques; $5 \mu$ m-thick sections were cut, stained with hematoxylin and eosin, and examined for residual tumour, radiotherapy-induced changes and other pathological features appropriate for the assessment of colorectal carcinoma specimens.

Following the surgery, the patients received adjuvant postoperative external beam radiotherapy and chemotherapy as per standard of care, if positive lymph nodes were found in the resected specimen.

\section{Statistical analysis}

Descriptive statistics were computed for demographic variables. Sensitivity, specificity, and positive and negative predictive values for the prediction of residual cancer were calculated, with 95\% CIs. These test performance characteristics were also calculated for assessment of nodal involvement. Kappa statistics measuring agreement beyond chance were calculated.

Factors predicting residual tumour were assessed from both the pre-BT EUS (proportion with stage T3 disease, proportion with nodal involvement, MWT) and the post-BT EUS (proportion down-staged, absolute reduction in MWT). Unpaired Student's $t$ tests were used for hypothesis testing involving continuous variables, and the $\chi^{2}$ test (or Fisher's Exact test where appropriate) was used for comparisons of proportions.

\section{RESULTS}

Eighteen patients underwent a post-BT EUS assessment for the presence of residual tumour and none had endoclips remaining at this assessment. The mean age $\pm \mathrm{SD}$ was $70 \pm 11$ years (range 52 to 93 years) and $78 \%$ of the patients were male. Before BT, EUS showed two patients (11\%) who were stage T2 (invading the muscularis propria but not into the serosa) and $16(11 \%)$ who were T3 (through the muscularis propria into the serosa and/or perirectal fat). Mean MWT was $14 \pm 3.4 \mathrm{~mm}$ on EUS. Five patients (28\%; $95 \%$ CI $7 \%$ to $49 \%$ ) had regional lymph nodes that were sonographically suspicious for malignant involvement (N1); $72 \%$ did not have significant regional lymphadenopathy (N0).

In all 18 patients, resected specimens were evaluated according to the aforementioned pathology protocol. After BT, pathological examination revealed that seven patients $(39 \%$; $95 \% \mathrm{CI}$ $16 \%$ to $61 \% ; \mathrm{P}=0.0006$ versus the $0 \%$ expected if BT was not able to sterilize the tumour) had no evidence of residual tumour (Figures 1 and 2). One additional patient had only carcinoma in situ. Three of the 11 patients $(27 \%)$ with residual tumour

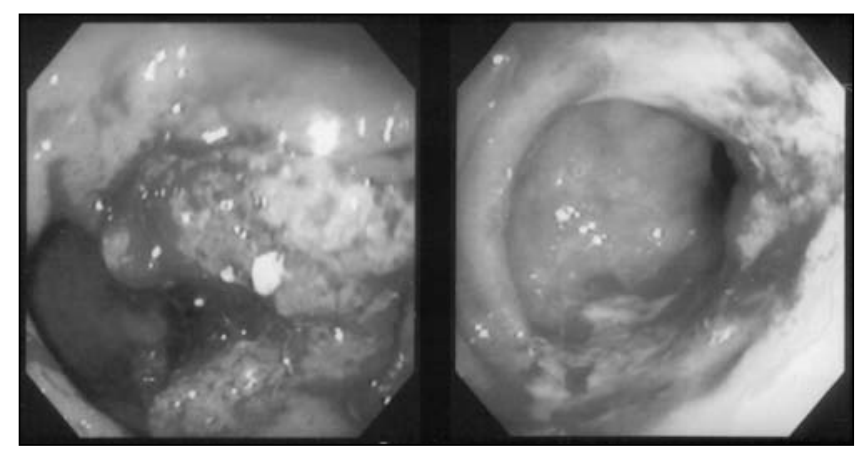

Figure 1) Endoscopic views of rectal adenocarcinoma pre- and postbrachytherapy in a patient with pathologically documented tumour sterilization (ie, no residual tumour), in the left and right panels, respectively. Significant radiation proctitis is seen in the right panel

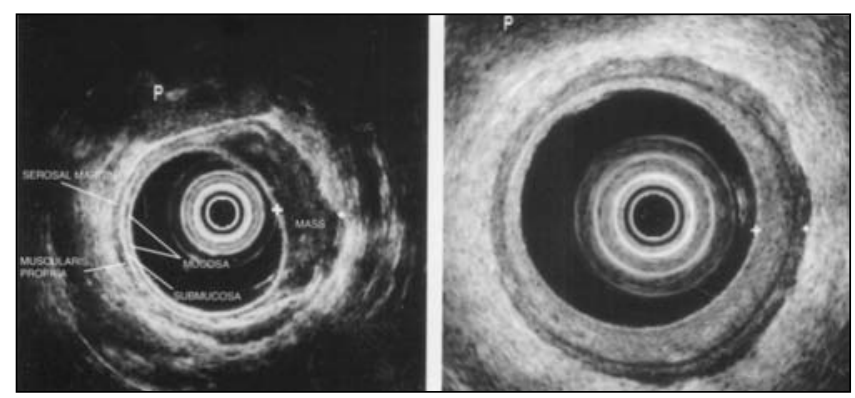

Figure 2) Radial endoscopic ultrasound (EUS) (7.5 MHz) images of rectal adenocarcinoma successfully 'sterilized' by brachytherapy (BT) and accurately diagnosed as such on post-BT EUS. Both images were taken from the same patient and at approximately the same distance from the anal verge. The left and right panels represent pre-and postBT EUS examinations, respectively. The two endosonographic mucosal layers, the hyperechoic submucosal layer, the hypoechoic muscularis propria layer, and the hyperechoic serosal margin are indicated in the left panel. The cancerous T3 mass is labelled in the left panel, as is the prostate $(P)$. The five endosonographic layers post-BT are thickened and blurred in the right panel

only had tiny foci of malignant cells, seen in selected blocks. One of the $18(5.6 \%)$ treated had residual T1 disease, one (5.6\%) had stage T2 disease and eight (44\%) had stage T3 disease. Significant downstaging occurred with significantly fewer patients having stage T3 disease after BT, in the surgical specimen ( $\mathrm{P}<0.001$; 95\% CI for difference: $17 \%$ to $72 \%)$. Mean MWT on EUS decreased from $14 \mathrm{~mm}$ to $9.2 \mathrm{~mm}$ $(\mathrm{P}<0.001)$. In contrast, there was no significant downstaging in nodal status. Eight of 18 patients (44\%) had positive nodes by pathology, after BT.

Eleven of 18 patients (61\%) underwent an APR for their distal carcinomas, including four $(36 \% ; 95 \%$ CI $8 \%$ to $65 \%$ ) who ultimately had no evidence of residual malignancy on pathological evaluation. Of the 11 with residual tumour, the degree of differentiation (grading) was reported as welldifferentiated in one, moderately differentiated in six and moderately to poorly differentiated in three patients; in one patient, the degree of differentiation could not be assessed reliably. One patient was found to have unexpected liver metastases at the time of surgery. Three patients (43\%) without residual tumour at pathology had documented extramural 


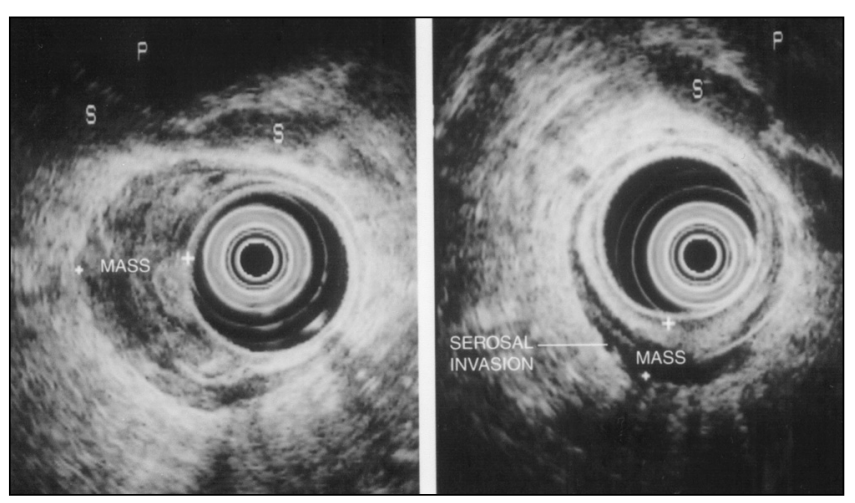

Figure 3) Radial endoscopic ultrasound (EUS) $(7.5 \mathrm{MHz})$ images of rectal adenocarcinoma which was successfully 'sterilized' by brachytherapy (BT), but interpreted as being unsuccessful by post-BT EUS (ie, false positive post-BT EUS). Both images were taken from the same patient and at approximately the same distance from the anal verge. The left and right panels represent pre-and post-BT EUS examinations, respectively. The EUS represented by the right panel was interpreted incorrectly as having residual tumour (MASS [Hypoechoic mass]), with invasion into the serosa (T3) as evidenced by pseudopodlike projections, illustrating the difficulty in these assessments. P Prostate; S Seminal vesicles

spread: two with regional lymph node involvement and the other with unexpected liver metastases found at the time of operation. One of the three (33\%) was detected to have nodes by EUS.

Median follow-up was 6.5 months from the time of recruitment (range two to 14 months). One patient died at nine months without local tumour recurrence; this patient had residual post-BT T3N1 disease by both EUS and pathology and had undergone an anterior resection with negative margins. No other recurrences have been documented.

Performance of post-BT EUS in predicting residual tumour Nine of the 11 patients with residual tumour were detected by EUS (sensitivity 82\%; 95\% CI 59\% to 100\%) (Figure 2). One of the two false negative exams was in a patient with carcinoma in situ. In contrast, there were five false positive EUS examinations (specificity 29\%; 95\% CI 0\% to 62\%) (Figure 3). Two of the five false positives (40\%) had mucin lakes and one had extensive necrosis. Most specimens without residual tumour had active inflammation. The corresponding positive and negative predictive values were $64 \%$ and $50 \%$, respectively, and the overall accuracy for predicting residual tumour was 61\% (95\% CI $39 \%$ to $84 \%$ ), with a corresponding poor kappa for agreement beyond chance $(0.11)$.

In eight patients (44\%; $95 \%$ CI $21 \%$ to $67 \%$ ), the pathological T-stage was exactly predicted on the post-BT EUS. Of the remaining 10 , seven ( $70 \%$; $95 \% \mathrm{CI} 42 \%$ to $98 \%$ ) were overstaged, but four of these $(40 \%)$ were still within one T-stage of the pathological stage. This comparison is summarized in Table 1, with exact matches bolded. Again, the corresponding kappa was poor for agreement beyond chance (0.11).

In the post-BT nodal assessments, there were four false negative and two false positive EUS examinations. These correspond to a sensitivity of $50 \%$ (95\% CI $15 \%$ to $85 \%$ ) and a specificity of $80 \%$ (95\% CI $55 \%$ to $100 \%)$.
TABLE 1

Comparison and correlation of tumour (T)-staging by post-brachytherapy endoscopic ultrasound (EUS) and by surgical pathology

\begin{tabular}{|c|c|c|c|c|c|c|c|}
\hline \multicolumn{8}{|c|}{ Pathology } \\
\hline & & T0 & T1 & T2 & T3 & T4 & TOTALS \\
\hline & TO & 2 & $1^{*}$ & 0 & 1 & 0 & 4 \\
\hline E & T1 & 1 & 0 & 0 & 1 & 0 & 2 \\
\hline $\mathbf{U}$ & T2 & 0 & $1^{\dagger}$ & 0 & 0 & 0 & 1 \\
\hline \multirow[t]{3}{*}{$\mathbf{S}$} & T3 & 4 & 0 & 1 & $6^{\ddagger}$ & 0 & 11 \\
\hline & T4 & 0 & 0 & 0 & 0 & 0 & 0 \\
\hline & Totals (\%) & 7 (39) & $2(11)$ & $1(5.6)$ & $8(44)$ & $0(0)$ & 18 \\
\hline
\end{tabular}

*This patient had only a small focus of tumour at pathology; ${ }^{\dagger}$ This patient actually had only carcinoma in situ at pathology; $¥$ Two of these six patients had only a small focus of tumour at pathology. The last row of the table shows the prevalence of different T-stages of disease as assessed by surgical pathology (the gold standard). Bolded results indicate complete agreement. TO indicates absence of residual tumour (tumour sterilization)

\section{Predictors of residual tumour}

A univariate comparison of baseline (eg, pretreatment) factors between patients with successful tumour 'sterilization' and those with residual tumour was performed. There was no statistically significant difference in age, prevalence of pretreatment stage T3 disease, prevalence of pretreatment nodal involvement or MWT (Table 2). Neither the downstaging rates nor the absolute reductions in MWT were significantly different in 'sterilized' patients versus those with residual tumour (Table 2). Patients with stage T3 disease and those who did not appear to be downstaged by EUS were more likely to ultimately have residual tumour after BT; the strength of these trends did not reach statistical significance. Also, proportionally, nodal disease was more prevalent in the sterilized group than in the group with residual disease; however, the CI of the difference was wide (Table 2). These negative exploratory analyses lack the statistical power to be definitive.

\section{DISCUSSION}

The accurate identification of patients with and without residual tumour after a BT protocol that promises a high tumour sterilization rate in rectal adenocarcinoma would be ideal and could, in theory, lead to less extensive surgical intervention. EUS is the logical test for this purpose, although external beam radiation-induced inflammation decreases the accuracy of the EUS assessments. In the present study, the inflammatory changes caused by BT were also a significant limitation, leading to a high false positive rate for EUS in predicting post-BT residual tumour. The sensitivity for predicting residual tumour was reasonable but the negative predictive value was still poor.

One potential limitation was our inability to arrange preoperative post-BT assessments on a significant number of patients enrolled in the BT study. There is no reason to believe that patients selected by these scheduling difficulties would bias the apparent EUS accuracy in either direction.

Although higher stage tumours, in theory, could have been more urgently operated on and consequently missed their postBT EUS, the high prevalence (89\%) of stage T3 lesions in our cohort suggests this did not occur.

Overstaging by EUS after BT was a significant problem (Table 1): the inflammation that extends to the serosal edge, 
TABLE 2

Comparison of baseline characteristics and changes in endoscopic ultrasound (EUS) appearance as a result of brachytherapy (BT) in patients with and without post-BT residual tumour

\begin{tabular}{|c|c|c|c|c|}
\hline & & $\begin{array}{c}\text { 'Sterile' specimen } \\
\text { (no tumour) } \\
n=7\end{array}$ & $\begin{array}{c}\text { Residual } \\
\text { tumour } \\
n=11\end{array}$ & $\begin{array}{l}\text { Difference } \\
(95 \% \mathrm{Cl})\end{array}$ \\
\hline Baseline factors & Age (years) & 72 & 69 & $3(-8$ to 15$)$ \\
\hline \multirow[t]{3}{*}{ assessed by pre-BT EUS } & Tumour stage T3 (\%) & 100 & 82 & $18(-5$ to 41$)$ \\
\hline & Lymph node stage N1 (\%) & 43 & 18 & 25 (-19 to 68$)$ \\
\hline & MWT (mm) & 14 & 14 & $0(-4$ to 4$)$ \\
\hline Changes due to BT & T-downstaged (\%) & 43 & 27 & $16(-30$ to 61$)$ \\
\hline assessed by EUS alone & Decrease in MWT (mm) & 5.0 & 5.0 & $0(-4$ to 4$)$ \\
\hline
\end{tabular}

MWT Maximum wall thickness by EUS. All comparisons $P>0.05$

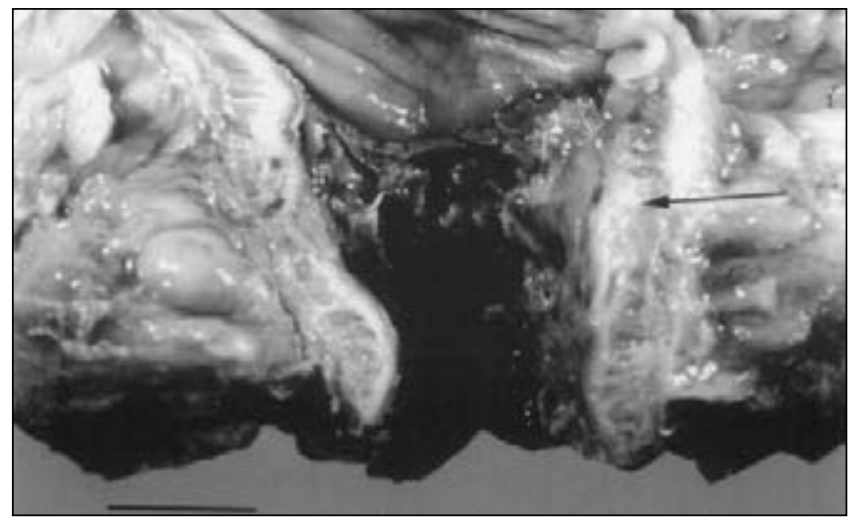

Figure 4) Distal end of resected specimen of rectum opened longitudinally in a patient without residual tumour at the surgery following brachytherapy, showing ulceration and permeation of the muscularis propria by amorphous mucus (arrow). Line indicates $2 \mathrm{~cm}$

producing an irregular margin with small pseudopod-like projections, will likely remain indistinguishable from serosal invasion. Disappointingly, even though these previously described artifacts (15) were taken into account in our EUS evaluation of these patients after BT, and despite a six-week average lag time between BT and EUS, there remained a significant number of false positive assessments. Clinically, however, false positives are preferable to false negatives.

Some post-BT EUS examinations in patients with complete tumour sterilization revealed focal hypoechoic masses, as demonstrated in Figure 3. The focal nature of this tailored conformal technique, which targets high doses of radiotherapy to those specific regions of the wall most involved by tumour, may lead to these more focal changes rather than the typical symmetrical diffuse changes seen with external beam radiotherapy. These masses mimicking recurrent tumour may represent focal edema from inflammation, or perhaps alternatively, necrosis or mucin lakes (Figures 4-6) as was seen pathologically in some specimens. The addition of deep tissue needle biopsy of endosonographically suspicious residual intramural masses, using curvilinear EUS and fine-needle aspiration (FNA) technique, is a possible future solution to this problem, with or without polymerase chain reaction to look for micrometastases. However, a negative cytology would still not completely exclude the presence of residual tumour.

One limitation of this study is the poor sensitivity of EUS for $\mathrm{N}$-staging. Nodal re-staging was not a primary endpoint of

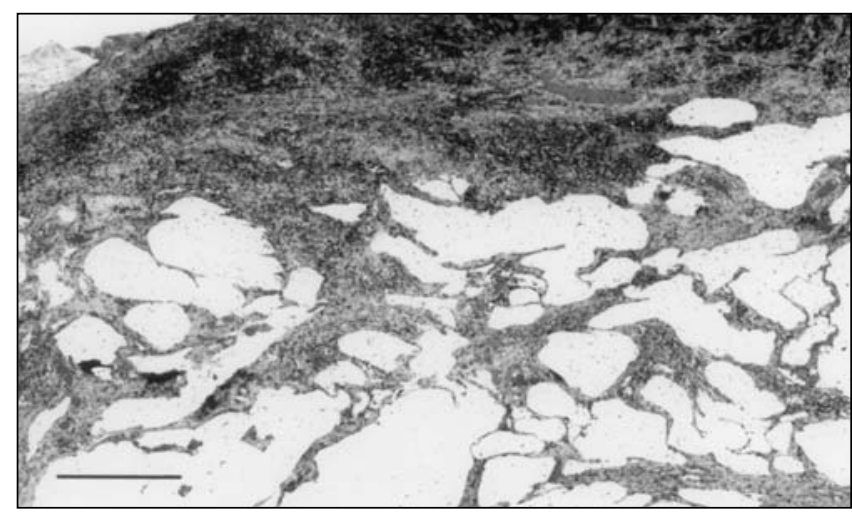

Figure 5) Low-power photomicrograph of the rectal wall from the same patient as in Figure 4 showing the interconnecting lakes of mucus in the muscularis propria. The ulcerated submucosa above is infiltrated by the dark inflammatory cells. Line indicates 500 um. Hematoxylin and eosin stain

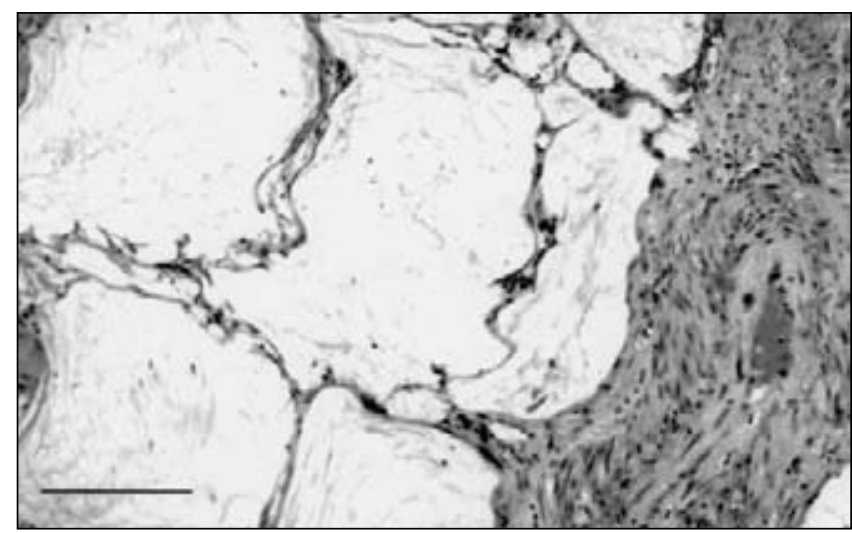

Figure 6) Medium-power photomicrograph showing pools of mucus infiltrating smooth muscle of muscularis propria, without evidence of carcinoma cells. Line indicates $200 \mu \mathrm{m}$. Hematoxylin and eosin stain

this particular study; we did not expect BT to alter lymph node status because of its limited depth of penetration and its lack of effect on $\mathrm{N}$-staging, as shown in previous studies (20). Furthermore, some of the missed lymph nodes are beyond the reach of the echoendoscope. The addition of EUS-guided FNA may have improved our results in terms of $\mathrm{N}$-staging.

In our clinical context, a high negative predictive value (true negatives as a proportion of all negative assessments) would be ideal to 'rule out' residual tumour. Because the residual tumour 
prevalence was relatively high $(61 \%)$, the negative predictive value was correspondingly poor $(50 \%)$, despite a reasonable sensitivity. Improvements in the specificity, and perhaps even the sensitivity, by mural FNA via EUS, possibly with polymerase chain reaction, as mentioned above, may improve the negative predictive value in the future.

\section{CONCLUSIONS}

Radial EUS assessment of residual rectal adenocarcinoma after high-dose rate conformal BT is inaccurate in terms of re-staging. Although it appears sensitive for detecting residual disease, the false positive rate remains high and the poor predictive values corresponding to this moderate prevalence of residual disease limit its utility in this setting. Further studies are needed to examine if these patients with complete histologic sterilization of their low-lying tumours could be candidates for sphinctersparing operations.

\section{REFERENCES}

1. Parker SL, Tong T, Bolden S, Wingo PA. Cancer statistics, 1996. CA Cancer J Clin 1996;46:5-27.

2. Krook JE, Moertel CG, Gunderson LL, et al. Effective adjuvant therapy for high risk rectal carcinoma. N Engl J Med 1991;324:709-15.

3. O'Connell MJ, Martenson JA, Wieand HS, et al. Improving adjuvant therapy for rectal cancer by combining protractedinfusion fluorouracil with radiation therapy after curative surgery. N Engl J Med 1994;331:502-7.

4. Tanum G, Wiig J, Andersen OK. Endoluminal radiotherapy for rectal tumors. Oncology 1998;55:521-4.

5. Mendenhall WM, Rout WR, Vauthey J-N, Haigh LS, Zlotecki RA, Copeland EM 3rd. Conservative treatment of rectal adenocarcinoma with endocavitary irradiation or wide local excision and postoperative irradiation. J Clin Oncol 1997; 15:3241-8.

6. Gerard JP, Baulieux J, Francois Y, et al. The role of radiotherapy in the conservative treatment of rectal carcinoma - the Lyon experience. Acta Oncologica 1998;37:253-8.
7. Farouk R, Nelson H, Gunderson LL. Aggressive multimodality treatment for locally advanced irresectable rectal cancer. Br J Surg 1997;84:741-9.

8. Gerard JP, Romestaing P, Ardiet JM, Momex F. Sphincter preservation in rectal cancer: Endocavitary radiation therapy. Semin Radiat Oncol 1998;8:13-23.

9. Kamikonya N, Izumi M, Tusboi K, Nogi K, Miyamoto Y, Nakao N. Results of preoperative high-dose-rate intraluminal brachytherapy for resectable rectal cancer. Nippon Igaku Hoshasen Gakkai Zasshi 1998;58:503-8.

10. Nishimaki T, Tanaka O, Ando N, et al. Evaluation of the accuracy of preoperative staging in thoracic esophageal cancer. Ann Thorac Surg 1999;68:2059-64.

11. Meneu-Diaz JC, Blazquez LA, Vicente E, et al. The role of multimodality therapy for resectable esophageal cancer. Am J Surg 2000;179:508-13.

12. Alexander EP, Lipman T, Harmon J, Wadleigh R. Aggressive multimodality therapy for stage III esophageal cancer: A phase I/II study. Ann Thorac Surg 2000;69:363-8.

13. Heriot AG, Grundy A, Kumar D. Preoperative staging of rectal carcinoma. Br J Surg 1999;86:17-28.

14. Thaler W, Watzka S, Martin F, et al. Preoperative staging of rectal cancer by endoluminal ultrasound vs. magnetic resonance imaging. Preliminary results of a prospective comparative study. Dis Colon Rectum 1994;37:1189-93.

15. Napoleon B, Pujol B, Berger F, Valette PJ, Gerard JP, Souquet JC. Accuracy of endosonography in the staging of rectal cancer treated by radiotherapy. Br J Surg 1991;78:785-8.

16. Giovannini M, Seitz JF, Thomas P, et al. Endoscopic ultrasonography for assessment of the response to combined radiation therapy and chemotherapy in patients with esophageal cancer. Endoscopy 1997;29:4-9.

17. Isenberg G, Chak A, Canto MI, et al. Endoscopic ultrasound in restaging of esophageal cancer after neoadjuvant chemoradiation. Gastrointest Endosc 1998;48:158-63.

18. Belliveau P, Vuong T, Michel R, et al. Conformal preoperative endorectal brachytherapy for patients with locally advanced rectal carcinoma. Clin Invest Med 2000;23:S101.

19. American Joint Committee on Cancer. Colorectal Cancer. Cancer Staging Manual. Philadelphia: Lippincott-Raven, 1997.

20. Yanagi H, Kusunoki M, Yamamura T. The effectiveness of preoperative intraluminal brachytherapy in preventing wall penetration and nodal involvement of rectal carcinomas. Surg Today 2000;30:410-5. 


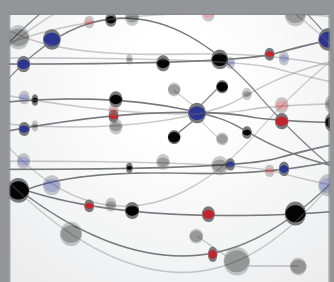

The Scientific World Journal
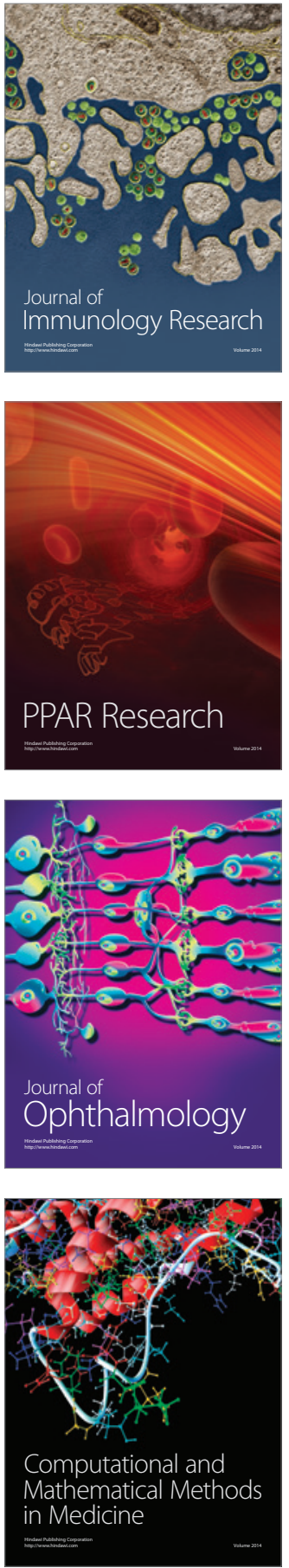

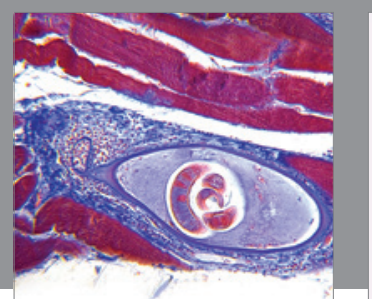

Gastroenterology Research and Practice

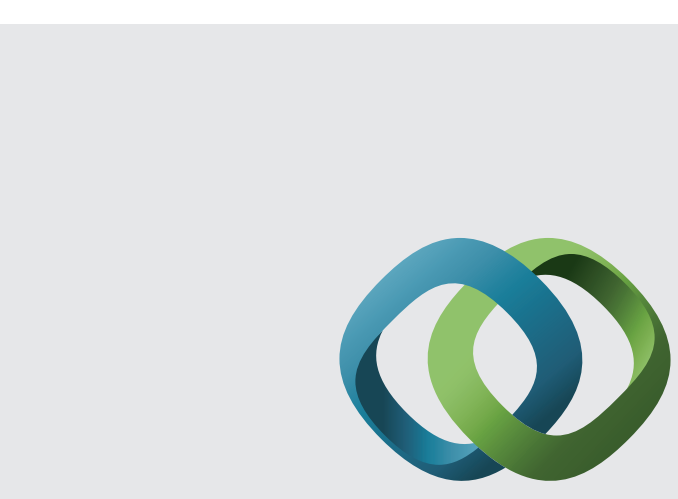

\section{Hindawi}

Submit your manuscripts at

http://www.hindawi.com
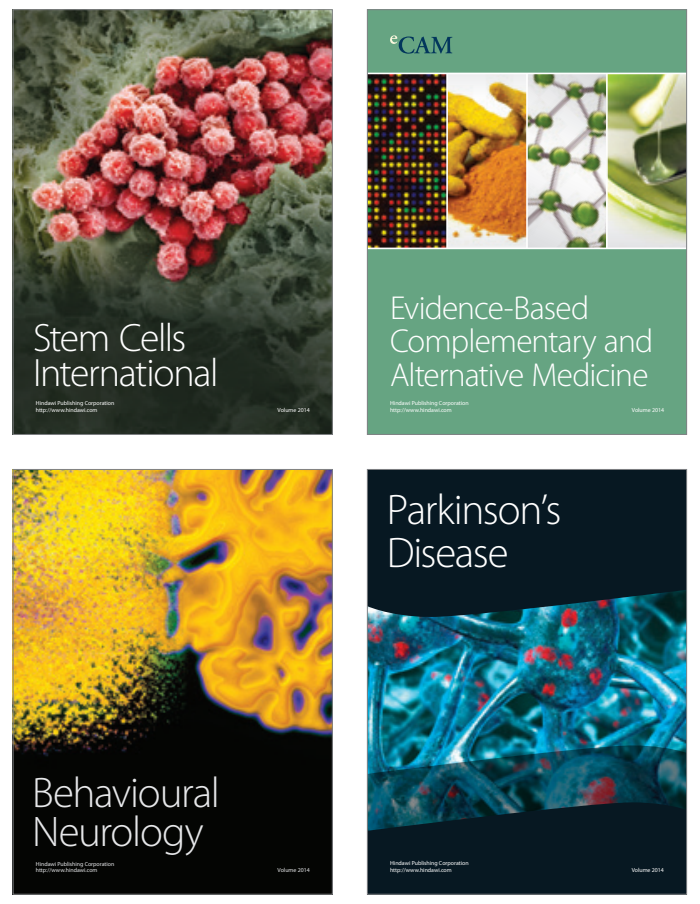
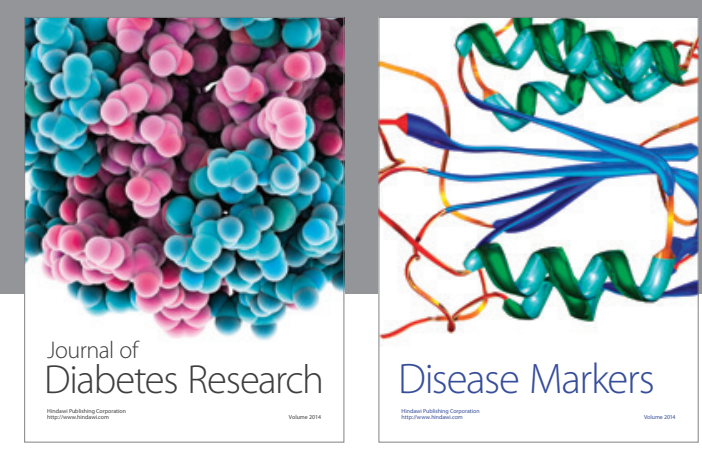

Disease Markers
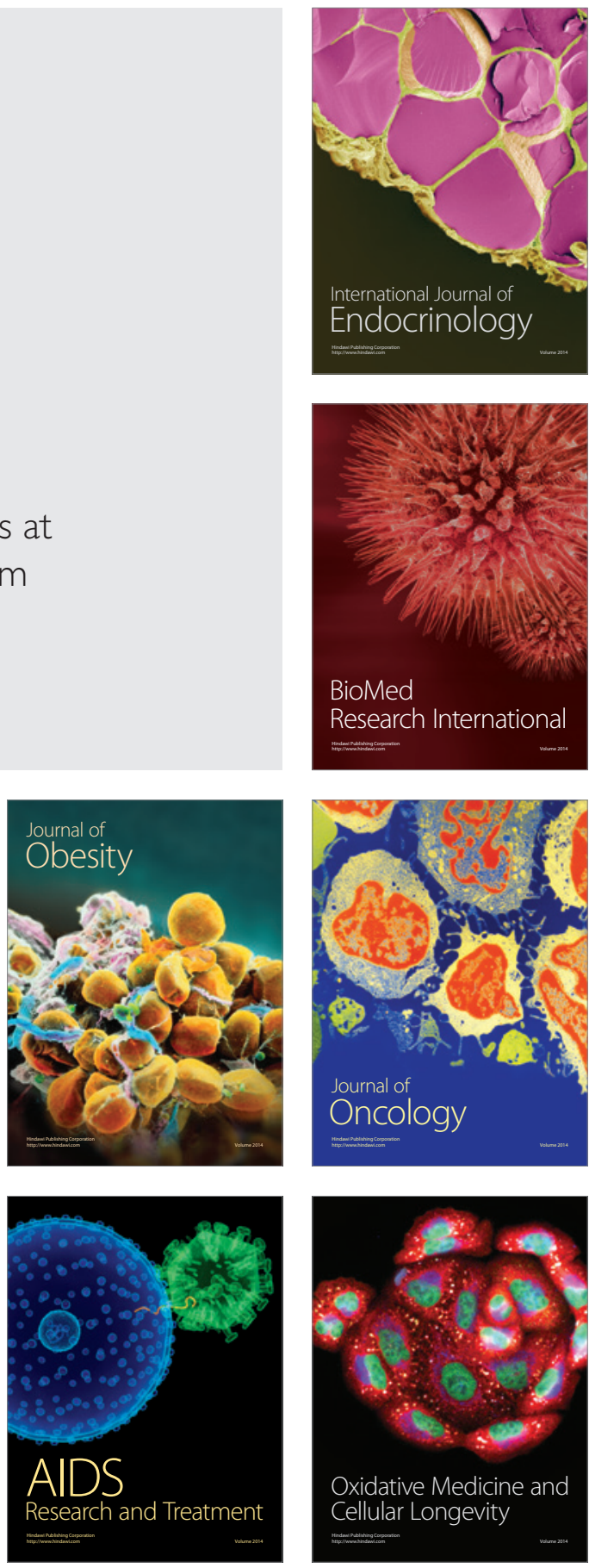